\title{
COMPARISON THEOREMS FOR A CLASS OF SELFADJOINT FOURTH ORDER DIFFERENTIAL EQUATIONS
}

\begin{abstract}
KURT KREITH
ABSTRACT. Equations of the form $\left(r(x) y^{\prime \prime}\right)^{\prime \prime}+p(x) y=0$ are considered under the assumption $r(x)>0$ and $p(x)>0$ in an interval $[\alpha, \infty)$. A polar coordinate transformation is used to study the effect of variations in the coefficients on the conjugate points of such equations.
\end{abstract}

1. Introduction. Oscillation properties of fourth order differential equations are frequently discussed in terms of the existence of conjugate points defined as follows: $\eta_{n}(\alpha)$ is the smallest $\beta>\alpha$ such that a nontrivial solution of the equation has $n+3$ zeros in $[\alpha, \beta]$ (multiple zeros being counted according to their multiplicities). For the case of equations of the form

$$
\left(r(x) y^{\prime \prime}\right)^{\prime \prime}+p(x) y=0
$$

with $r(x)$ and $p(x)$ positive in [ $\alpha, \infty)$, Leighton and Nehari [1] have shown that $\eta_{n}(\alpha)$ is attained by a solution of (1) having a triple zero at $\alpha$ and $n$ simple zeros in $(\alpha, \beta]$. In $\S 10$ of [1] a number of comparison theorems are established which describe how such conjugate points vary with changes in the coefficients of (1.1).

The purpose of this paper is to present such comparison theorems in the more general context of the rotation theory developed in [2]. This will enable us to simplify some arguments and also to fill a gap in the proof of Theorem 10.2 of [1]. (The nature and consequences of this gap are discussed in $\$ 3$ below.)

Instead of studying such equations in the form (1.1), we shall write them as second order systems of the form

$$
y^{\prime \prime}=b(x) z, \quad z^{\prime \prime}=c(x) y
$$

where $b(x)$ and $-c(x)$ are assumed positive and continuous in an interval $[\alpha, \infty]$. The substitutions

$$
b=1 / r ; \quad c=-p ; \quad z=r y^{\prime \prime}
$$

clearly transform (1.2) into (1.1). The advantage of the latter form is that it enables us to interpret conjugate points and oscillation properties in terms of the rotation theory developed in [2]. Some basic ideas and results from this

Received by the editors August 6, 1976.

AMS (MOS) subject classifications (1970). Primary 34C10.

Key words and phrases. Conjugate points, rotation theory, principal solution, phase function. 
theory are presented in the following section. The comparison theorems are established in $\$ 3$ and applied to fourth order equations in $\$ 4$.

2. Rotation theory. The solutions of the system (1.2) may be regarded as trajectories $(z(x), y(x))$ in the $z, y$-plane. Rotation properties of such trajectories are expressed in terms of functions $\rho(x)$ and $\theta(x)$ defined by

$$
\rho^{2}=z^{2}+y^{2} ; \quad \tan \theta=y / z
$$

which transforms (1.2) into

$$
\begin{gathered}
\rho^{\prime \prime}=\rho \theta^{\prime 2}+(b(x)+c(x)) \frac{y z}{\rho}, \\
\left(\rho^{2} \theta^{\prime}\right)^{\prime}=b(x) z^{2}-c(x) y^{2} .
\end{gathered}
$$

Defining $\varphi(x)=\rho^{2} \theta^{\prime}$, the " area function" $\varphi$ denotes the rate at which area is being swept out by the radius vector $(z(x), y(x))$. Our hypotheses $b(x)>0$ and $-c(x)>0$ assure that for any nontrivial solution, $\varphi^{\prime}(x) \geqslant 0$ in $[\alpha, \infty]$ and positive except perhaps for a single $x$. Since the initial conditions $y(\alpha)=z(\alpha)=0$ imply $\varphi(\alpha)=0$, they also imply that $\varphi(x)>0$ for all $x>\alpha$. Thus the hypotheses $b>0, c<0$ and $y(\alpha)=z(\alpha)=0$ assure that $\theta^{\prime}(x)>0$ for all $x>\alpha$, and it is this monotone character of the phase function $\theta(x)$ which underlies much of the analysis to follow.

The fact that conjugate points with respect to (1.1) are attained by solutions having a triple zero at $x=\alpha$ underlies our special interest in principal solutions $Z(x ; \alpha), \quad Y(x ; \alpha)$ of $(1.2)$ satisfying $Z(\alpha)=Y(\alpha)=Y^{\prime}(\alpha)=0$. Using an upper case $\Theta(x ; \alpha)$ to represent the phase functions of such principal solutions, we note that the solutions of $\Theta(x ; \alpha) \equiv 0(\bmod \pi)$ are precisely the conjugate points of $\alpha$ (with respect to (1.2)).

We shall also have occasion to refer to $\theta_{0}$-principal solutions of (1.2) satisfying

$$
Z(\alpha)=Y(\alpha)=0 ; \quad \lim _{x \downarrow \alpha} \frac{Y^{\prime}(x)}{Z^{\prime}(x)}=\tan \theta_{0} ; \quad\left(\sqrt{Z^{2}+Y^{2}}\right)^{\prime}(\alpha)=1
$$

and denoted by $Z\left(x ; \alpha, \theta_{0}\right), Y\left(x ; \alpha, \theta_{0}\right)$. For any $\theta_{0}$-principal solution the area function $\Phi(x)$ satisfies $\Phi(\alpha)=0$ and $\Phi(x)>0$ for $x>\alpha$. A specific result from [2] which will be required below is the following monotonicity theorem.

2.1. Theorem. Suppose $\alpha \leqslant \alpha_{1}<\alpha_{2}$ and that $Z_{i}(x), Y_{i}(x)=Z_{i}\left(x ; \alpha_{i}, \theta_{0}\right)$, $Y_{i}\left(x ; \alpha_{i}, \theta_{0}\right)$ are $\theta_{0}$-principal solutions for $i=1,2$. If the corresponding phase functions satisfy

$$
k \pi \leqslant \Theta_{1}\left(\alpha_{2}\right)-\Theta_{2}\left(\alpha_{2}\right)<(k+1) \pi
$$

for some integer $k$, then

$$
k \pi<\Theta_{1}(x)-\Theta_{2}(x)<(k+2) \pi
$$

for all $x>\alpha_{2}$.

Proof. See [2, Theorem 3.1]. 
Another required result is the following.

2.2. Theorem. Let $Z(x), Y(x)=Z\left(x ; \alpha, \theta_{0}\right), Y\left(x ; \alpha, \theta_{0}\right)$ denote the $\theta_{0}$-principal solution of (1.2) and let $z(x), y(x)$ be any nontrivial solution of (1.2) satisfying $\theta(\alpha)=\theta_{0}+k \pi$ and $\varphi(\alpha)>0$. Then for any $x>\alpha, k \pi<\theta(x)-$ $\Theta(x)<(k+1) \pi$.

Proof. Suppose to the contrary that there exists a $\gamma>\alpha$ such that $\theta(\gamma) \equiv \Theta(\gamma)(\bmod \pi)$. Then by Lemma 2.1 of [2] there exist nontrivial constants $\lambda, \mu$ such that

$$
\tilde{z}(x)=\lambda z(x)+\mu Z(x) ; \quad \tilde{y}(x)=\lambda y(x)+\mu Y(x)
$$

satisfies (1.2) and $\tilde{z}(\gamma)=\tilde{y}(\gamma)=0$. The corresponding area function therefore satisfies $\tilde{\varphi}(\gamma)=0$, and since $\tilde{\varphi}^{\prime}(x)>0$, we have $\tilde{\varphi}(\alpha)<0$. On the other hand, a direct computation shows that

$$
\begin{aligned}
\tilde{\varphi}(x)= & \lambda^{2} \varphi(x)+\mu^{2} \Phi(x) \\
& +\lambda \mu\left[Y^{\prime}(x) z(x)+y^{\prime}(x) Z(x)-y(x) Z^{\prime}(x)-Y(x) z^{\prime}(x)\right] .
\end{aligned}
$$

As explained in [2], we may assume without loss of generality that $\theta_{0}=0$, so that the initial conditions imply

$$
\Phi(\alpha)=Y(\alpha)=Y^{\prime}(\alpha)=Z(\alpha)=y(\alpha)=0 .
$$

But this yields $\tilde{\varphi}(\alpha)=\lambda^{2} \varphi(\alpha) \geqslant 0$ and this contradiction establishes the theorem .

The next preparatory result is one which relates the values of the phase function $\Theta(x)$ of a principal solution $Z(x ; \alpha), Y(x ; \alpha)$ with the phase functions of principal solutions initiated at various conjugate points of $\alpha$. We formulate it in a slightly more general context by referring to a given $\theta_{0}$-principal solution.

2.3. Theorem. Let $Z\left(x ; \alpha, \theta_{0}\right), Y\left(x ; \alpha, \theta_{0}\right)$ denote a $\theta_{0}$-principal solution (1.2) whose phase function satisfies $\Theta\left(\alpha_{k}\right)=\theta_{0}+(2 k-1) \pi$ for $k=$ $1,2, \ldots, n$. Let

$$
Z_{k}\left(x ; \alpha_{k}, \theta_{0}+(2 k-1) \pi\right), \quad Y_{k}\left(x ; \alpha_{k}, \theta_{0}+(2 k-1) \pi\right)
$$

denote a family of $\theta_{0}+(2 k-1) \pi$ principal solutions with phase functions $\Theta_{k}(x)$. If $\beta_{1}, \ldots, \beta_{n-1}$ are defined by $\Theta_{k}\left(\beta_{k}\right)=\theta_{0}+2 k \pi$, then $\beta_{k}<\alpha_{k+1}$ for $k=1, \ldots, n-1$.

Proof. By Theorem 2.2 we have

$$
0<\Theta(x)-\Theta_{k}(x)<\pi
$$

for all $k \geqslant 1$ and $x>\alpha_{k}$. In particular,

$$
0<\Theta\left(\alpha_{k+1}\right)-\Theta_{k}\left(\alpha_{k+1}\right)=\theta_{0}+(2 k+1) \pi-\Theta_{k}\left(\alpha_{k+1}\right)<\pi
$$

so that

$$
\Theta_{k}\left(\alpha_{k+1}\right)>\theta_{0}+2 k \pi=\Theta_{k}\left(\beta_{k}\right) .
$$

In the special case $\theta_{0}=0$, the $\alpha_{k}$ are just the $2 k-1$ conjugate points of $\alpha$. 
We may also define iterative conjugate points of $\alpha$ by

$$
\eta^{(1)}(\alpha)=\eta_{1}(\alpha) ; \quad \eta^{(k+1)}(\alpha)=\eta_{1}\left(\eta^{(k)}(\alpha)\right) \quad(k=1,2, \ldots)
$$

with the understanding that $\eta_{1}(\infty)=\infty$. By Theorem 2.3 and successive applications of Theorem 2.1, we have $\eta^{(k+1)}(\alpha) \leqslant \beta_{k}$. This yields the following important estimate.

2.4. Corollary. If $k>1$ and $\eta_{2 k-1}(\alpha)<\infty$, then

$$
\eta^{(k)}(\alpha)<\eta_{2 k-1}(\alpha) \text {. }
$$

As a final preparatory result we note the following

2.5. Lemma. Let $Z_{1}(x ; \alpha), Y_{1}(x ; \alpha)$ denote a principal solution of $(1.2)$ and $Z_{2}\left(x ; \alpha, \theta_{0}\right), Y_{2}\left(x ; \alpha, \theta_{0}\right)$ denote a $\theta_{0}$-principal solution with $k \pi<\theta_{0}<(k+$ 1) $\pi$. Then for all $x>\alpha, k \pi<\Theta_{2}(x)-\Theta_{1}(x)<(k+1) \pi$.

Proof. If for some $\gamma>\alpha, \Theta_{2}(\gamma) \equiv \Theta_{1}(\gamma)(\bmod \pi)$ then there exist nontrivial constants $\lambda, \mu$ such that

$$
\tilde{z}(x)=\lambda Z_{1}(x)+\mu Z_{2}(x) ; \quad \tilde{y}(x)=\lambda Y_{1}+\mu Y_{2}(x)
$$

satisfies (1.2) and $\tilde{z}(\alpha)=\tilde{y}(\alpha)=0$. Since the corresponding area function satisfies $\tilde{\varphi}^{\prime}(x)>0$ for $x>\alpha$ and $\tilde{\varphi}(\gamma)=0$, we have $\tilde{\varphi}(\alpha)<0$. On the other hand

$$
\begin{aligned}
\tilde{\varphi}(x)= & \lambda^{2} \Phi_{1}(x)+\mu^{2} \Phi_{2}(x) \\
& +\lambda \mu\left[Y_{2}^{\prime}(x) Z_{1}(x)-Y_{1}^{\prime}(x) Z_{2}(x)-Y_{1}(x) Z_{2}^{\prime}(x)-Y_{2}(x) Z_{1}^{\prime}(x)\right]
\end{aligned}
$$

yields $\tilde{\varphi}(\alpha)=0$ which is the desired contradiction.

3. Comparison theorems. We shall consider the following systems of the form (1.2):

$$
y_{1}^{\prime \prime}=b(x) z_{1} ; \quad z_{1}^{\prime \prime}=c(x) y_{1}
$$

with conjugate points denoted by $\eta$;

$$
y_{2}^{\prime \prime}=b(x) z_{2} ; \quad z_{2}^{\prime \prime}=C(x) y_{2}
$$

with $C(x) \leqslant c(x)<0$ and conjugate points denoted by $\xi$;

$$
y_{3}^{\prime \prime}=B(x) z_{3} ; \quad z_{3}^{\prime \prime}=c(x) y_{3}
$$

with $B(x) \geqslant b(x)>0$ and conjugate points denoted by $\nu$; and

$$
y_{4}^{\prime \prime}=B(x) z_{4} ; \quad z_{4}^{\prime \prime}=C(x) y_{4}
$$

with $C(x) \leqslant c(x)<0, B(x) \geqslant b(x)>0$, and conjugate points denoted by $\zeta$. The following comparison theorem is established in [1] .

3.1. ThEOREM. If $C(x) \leqslant c(x)<0$ in $[\alpha, \infty)$, then

$$
\xi_{1}(\alpha) \leqslant \eta_{1}(\alpha)
$$

An immediate consequence of this and Theorem 2.1 is

3.2. Corollary. If $C(x) \leqslant c(x)<0$ in $[\alpha, \infty)$, then 


$$
\xi^{(k)}(\alpha) \leqslant \eta^{(k)}(\alpha)
$$

for all positive integers $k$.

In passing, we note that the techniques of [1] or Corollary 3.2 enable us to sharpen slightly the estimate (10.10) of [1].

3.3. TheOREM. If $C(x) \leqslant c(x)<0$ in $[\alpha, \infty)$, then

$$
\xi_{k}(\alpha) \leqslant \eta_{2 k-1}(\alpha), \quad k=1,2, \ldots,
$$

with strict inequality if $k>1$ and $\eta_{2 k-1}(\alpha)<\infty$.

Proof. It follows from Theorem 2.1 that $\xi_{k}(\alpha) \leqslant \xi^{(k)}(\alpha)$, with strict inequality if $k>1$ and $\xi^{(k)}(\alpha)<\infty$. By Corollaries 2.4 and 3.2 we therefore have

$$
\xi_{k}(\alpha) \leqslant \xi^{(k)}(\alpha) \leqslant \eta^{(k)}(\alpha) \leqslant \eta_{2 k-1}(\alpha)
$$

with strict inequality if $k>1$ and $\eta_{2 k-1}(\alpha)<\infty$.

The difficulties in [1] arise in connection with the reciprocal equations (10.8) and (10.9). We circumvent these by defining $\tilde{\eta}_{k}(\alpha)$ with respect to (3.1) by the equations

$$
\tilde{\Theta}_{1}\left(\tilde{\eta}_{k}(\alpha)\right)=(k+1 / 2) \pi
$$

where $\tilde{\Theta}_{1}(x)$ is the phase function associated with the $\pi / 2$-principal solution

$$
Z_{1}(x ; \alpha, \pi / 2) ; \quad Y_{1}(x ; \alpha, \pi / 2)
$$

and the understanding that $\tilde{\eta}_{k}(\alpha)=\infty$ if $\Theta_{1}(x)<\left(k+\frac{1}{2}\right) \pi$ for all $x$. The $\tilde{\xi}_{k}(\alpha), \tilde{v}_{k}(\alpha), \tilde{\zeta}_{k}(\alpha)$ are analogously defined by $\tilde{\Theta}_{2}(x), \tilde{\Theta}_{3}(x), \tilde{\Theta}_{4}(x)$ with respect to (3.2), (3.3), (3.4). For these reciprocal conjugate points a virtually identical proof yields the following analogue of Theorem 3.3.

3.4. ThEOREM. If $0<b(x) \leqslant B(x)$ in $[\alpha, \infty)$, then

$$
\tilde{\nu}_{k}(\alpha) \leqslant \tilde{\eta}_{2 k-1}(\alpha), \quad k=1,2, \ldots,
$$

with strict inequality if $k>1$ and $\tilde{\eta}_{2 k-1}(\alpha)<\infty$.

The gap in the proof of Theorems 10.2 and 10.3 of [1] lies in the implicit but unwarranted assumption that (in our notation) $\nu_{k}(\alpha)=\tilde{\nu}_{k}(\alpha)$. In the absence of such an identity, a more detailed argument is required to relate to $\eta_{j}(\alpha)$ and $\nu_{k}(\alpha)$.

3.5. THEOREM. If $0<b(x) \leqslant B(x)$ in $[\alpha, \infty)$, then $\nu_{k-1}(\alpha) \leqslant \eta_{2 k}(\alpha)$ with strict inequality if $\eta_{2 k}(\alpha)<\infty$.

Proof. Assuming $\eta_{2 k}(\alpha)<\infty$, we write $\beta=\eta_{2 k}(\alpha)$ so that $\Theta_{1}(\beta)=2 k \pi$. By Lemma $2.5, \tilde{\Theta}_{1}(\beta)>2 k \pi$ so that $\tilde{\eta}_{2 k-1}(\alpha)<\beta$. By Theorem 3.4, $\tilde{v}_{k}(\alpha)<$ $\beta$ so that $\tilde{\Theta}_{3}(\beta)>\left(k+\frac{1}{2}\right) \pi$. Another application of Lemma 2.5 yields

$$
\Theta_{3}(\beta)>\left(k-\frac{1}{2}\right) \pi>(k-1) \pi,
$$

so that $\nu_{k-1}(\alpha)<\beta$ as was to be shown. 
We remark that this result is slightly weaker than the inequality $\nu_{k}(\alpha)<$ $\eta_{2 k}(\alpha)$ asserted in Theorem 10.3 of [1].

There remains the problem of comparing the $\eta_{j}$ with $\zeta_{k}$.

3.6. TheOREM. If $0<b(x) \leqslant B(x)$ and $C(x)<c(x)<0$ in $[\alpha, \infty)$, then $\zeta_{k}(\alpha) \leqslant \eta_{4 k}(\alpha)$ with inequality if $\eta_{4 k}(\alpha)<\infty$.

Proof. By Theorem 3.5, $\eta_{4 k}(\alpha) \geqslant \nu_{2 k-1}(\alpha)$. Applying Theorem 3.3 to the case where $b(x)$ is replaced by $B(x)$ yields $\nu_{2 k-1}(\alpha) \geqslant \zeta_{k}(\alpha)$. These combine to give the desired result.

4. Fourth order equations. The transformations

$$
\begin{array}{ll}
b(x)=1 / r(x), & c(x)=-p(x), \\
B(x)=1 / R(x), & C(x)=-P(x),
\end{array}
$$

convert (3.1)-(3.4) into

$$
\begin{aligned}
& \left(r(x) y^{\prime \prime}\right)^{\prime \prime}+p(x) y=0 \\
& \left(r(x) y^{\prime \prime}\right)^{\prime \prime}+P(x) y=0 \\
& \left(R(x) y^{\prime \prime}\right)^{\prime \prime}+p(x) y=0 \\
& \left(R(x) y^{\prime \prime}\right)^{\prime \prime}+P(x) y=0
\end{aligned}
$$

respectively, with conjugate points $\eta_{k}(\alpha), \xi_{k}(\alpha), \nu_{k}(\alpha)$, and $\zeta_{k}(\alpha)$. These equations have reciprocals

$$
\begin{aligned}
& \left(\frac{1}{p(x)} z^{\prime \prime}\right)^{\prime \prime}+\frac{1}{r(x)} z=0 \\
& \left(\frac{1}{P(x)} z^{\prime \prime}\right)^{\prime \prime}+\frac{1}{r(x)} z=0 \\
& \left(\frac{1}{p(x)} z^{\prime \prime}\right)^{\prime \prime}+\frac{1}{R(x)} z=0 \\
& \left(\frac{1}{P(x)} z^{\prime \prime}\right)^{\prime \prime}+\frac{1}{R(x)} z=0
\end{aligned}
$$

with conjugate points $\tilde{\eta}_{k}(\alpha), \tilde{\xi}_{k}(\alpha), \tilde{\nu}_{k}(\alpha)$ and $\tilde{\zeta}_{k}(\alpha)$, respectively. The theorems of $\$ 3$ are readily translated to apply to these equations. In the case of positive coefficients, all these equations have the property that if one solution is oscillatory at $x=\infty$, then all solutions are oscillatory. Thus, they can be classified as oscillatory or nonoscillatory depending on whether or not some solution is oscillatory. With this observation, the results of $\$ 3$ immediately imply the following.

4.1. Theorem. Suppose $R(x) \geqslant r(x)>0$ and $p(x) \geqslant P(x)>0$ in $[\alpha, \infty)$. If (4.1) is oscillatory, so are (4.2)-(4.4) and (4.1')-(4.4'). If (4.2) is oscillatory, so are (4.4), $\left(4.2^{\prime}\right)$, and $\left(4.4^{\prime}\right)$. If (4.3) is oscillatory, so are $(4.4),\left(4.3^{\prime}\right)$, and $\left(4.4^{\prime}\right)$.

This result establishes the validity of Theorem 10.4 of [1]. 


\section{REFERENCES}

1. W. Leighton and Z. Nehari, On the oscillation of solutions of self-adjoint linear differential equations of the fourth order, Trans. Amer. Math. Soc. 89 (1958), 325-377.

2. K. Kreith, Rotation properties of a class of second order differential systems, J. Differential Equations 17 (1975), 395-405.

Department of Mathematics, University of California, Davis, California 95616 